\title{
Simulation of vertical tidal turbine based on OpenFOAM and influence of inlet turbulence
}

\author{
Yun-ya Liu $^{1, *}, Y u$-chenYang ${ }^{2}$, and $Y a$-wen Yang ${ }^{1}$ \\ ${ }^{1}$ School of Oil \& Natural Gas Engineering, Southwest Petroleum University, Chengdu 610500, China \\ ${ }^{2}$ School of Mechanical Engineering, Southwest Petroleum University, Chengdu 610500, China
}

\begin{abstract}
This paper first introduces the basic theory of CFD method, including basic control equations, finite volume method, control equation solving algorithm and turbulence model selection. Second, based on OpenFOAM, an open-source fluid mechanics software, a numerical simulation method of vertical axis tidal turbine was proposed by using $\mathrm{k}-\omega$ SST turbulence model and PIMPLE algorithm. The hydrodynamic characteristics of the vertical axis turbine were studied, and the calculation results were compared with experiments. The higher consistency proves the feasibility of the numerical simulation method proposed in this paper. Finally, the influence of inlet turbulence on numerical simulation was explored, and a set of effective CFD simulation strategies was concluded, which provided a valuable reference for future CFD simulation and research on vertical axis tidal turbines.
\end{abstract}

\section{Introduction}

The constant exploitation of fossil fuels has caused environmental problems to become increasingly prominent. With the global advocacy of low-carbon emission reduction, renewable energy has become the focus of attention of all countries in the world. As a kind of renewable energy, tidal energy has the characteristics of high energy density, strong predictability, and stable load, so it is widely concerned by scholars at home and abroad. The research theories and methods of tidal turbines mainly come from the evolution of wind turbine technology or marine propeller technology. At present, research methods of tidal turbines are mainly divided into four categories: flow tube method, vortex method, experimental method and viscous CFD method.

With the rapid development of computer technology, CFD numerical simulation method has become an efficient and convenient means. So far, the CFD method has achieved more results in the study of vertical axis turbine. Nabavi[1] used Fluent software to simulate vertical axis turbine and compared them with experimental values; Gebreslassie et al.[2] simulated the Fluent method. For the vertical axis turbines placed in series, the conclusion is that the smaller longitudinal distance will cause a large loss of downstream tidal turbine energy. Li Zhichuan [3] used CFX to study the effects of different influencing factors of vertical axis turbines on the hydrodynamic performance of tidal turbines.

*Corresponding author: andrea_liuyy@163.com 
In summary, until now, CFD research on tidal turbines is basically based on commercial software such as CFX and FLUENT. From the perspective of the selection of CFD numerical models, apart from using commercial software, CFD numerical models can also have the following three options: First, develop your own CFD numerical simulation calculation code and write your own program for calculation. Second, based on the existing code, add a program that can solve practical problems to implement CFD calculation. Third, the source code of the open source code is used for calculation. Of these three methods, the first one is inappropriate, and the development of new CFD calculation codes requires solid mathematical knowledge. It also requires a lot of manpower and material resources and huge financial support; The second method needs to be implemented based on existing code. Therefore, this paper adopts the third method and chooses OpenFOAM, a CFD calculation software based on open source code.

This paper first proposes a numerical simulation method based on OpenFOAM to study the vertical axis turbine and verify the feasibility of this method. Compared with other commercial software, the advantage of this method is that it has higher solving efficiency and greater flexibility. According to the needs of users, it can build a solver and optimize the corresponding discrete method based on the original code. This achieves the optimization algorithm, saving time and cost. In the end, the irrelevance verification of the inlet turbulence degree is carried out in this paper, which provides reference and guidance for the future CFD simulation and research on the vertical axis tidal power turbine.

\section{The basic theory of CFD method}

\subsection{Basic control equations}

In general, the movement of fluid must satisfy the three basic laws: the law of conservation of mass, the law of conservation of momentum, and the law of conservation of energy. The three basic laws of conservation of the fluid are the continuous equation, momentum equation and energy equation in the flow of fluid.

The continuity equation of incompressible fluid is as follows:

$$
\operatorname{div} \boldsymbol{U}=0
$$

For incompressible fluids, the momentum equation is a generalized Navier-Stokes equation. If the components of the velocity vector $\mathrm{U}$ in the $x, y$, and $z$ directions in the geodetic coordinate system are denoted by $u, v, w$ respectively, the momentum equations of the incompressible fluid in the three coordinate directions can be expressed as:

$$
\begin{aligned}
& \frac{\partial u}{\partial t}+\operatorname{div}(u \boldsymbol{U})=f_{x}-\frac{1}{\rho} \frac{\partial p}{\partial x}+v \operatorname{div}(\operatorname{grad} u) \\
& \frac{\partial v}{\partial t}+\operatorname{div}(v \boldsymbol{U})=f_{y}-\frac{1}{\rho} \frac{\partial p}{\partial y}+v \operatorname{div}(\operatorname{grad} v) \\
& \frac{\partial w}{\partial t}+\operatorname{div}(w \boldsymbol{U})=f_{z}-\frac{1}{\rho} \frac{\partial p}{\partial z}+v \operatorname{div}(\operatorname{grad} w)
\end{aligned}
$$

Among them, $f_{\mathrm{x}}, f_{\mathrm{y}}, f_{\mathrm{z}}$ are the components of the mass force in the $x, y$, and $z$ directions, $p$ is the pressure, and $v$ is the fluid motion viscosity coefficient.

In the flow analysis, for the convenience of solving, the time-averaging method is often used to introduce the pulsation of the flow. For example, the turbulent flow velocity $U$ of the fluid is regarded as the sum of the average flow velocity $\bar{U}$ and the instantaneous pulsation velocity $U^{\prime}$.

$$
\boldsymbol{U}=\overline{\boldsymbol{U}}+\boldsymbol{U}^{\prime}
$$




$$
\overline{\boldsymbol{U}}=\frac{1}{\Delta t} \int_{t}^{t+\Delta t} \boldsymbol{U} d t
$$

The same physical quantities $\mathrm{u}, \mathrm{v}, \mathrm{w}, \mathrm{p}$ can be expressed as:

$$
u=\bar{u}+u^{\prime} \quad v=\bar{v}+v^{\prime} \quad w=\bar{w}+w^{\prime} \quad p=\bar{p}+p^{\prime}
$$

Substituting the above physical quantities into the momentum equation and the continuity equation is available:

$$
\begin{gathered}
\operatorname{div} \overline{\boldsymbol{U}}=0 \\
\frac{\partial \bar{u}}{\partial t}+\operatorname{div}(\bar{u} \overline{\boldsymbol{U}})=\overline{f_{x}}-\frac{1}{\rho} \frac{\partial \bar{p}}{\partial x}+v \cdot \operatorname{div}(\operatorname{grad} \bar{u})+\left[-\frac{\partial \overline{u^{\prime 2}}}{\partial x}-\frac{\partial \overline{u^{\prime} v^{\prime}}}{\partial y}-\frac{\partial \overline{u^{\prime} w^{\prime}}}{\partial z}\right] \\
\frac{\partial \bar{v}}{\partial t}+\operatorname{div}(\bar{v} \overline{\boldsymbol{U}})=\overline{f_{y}}-\frac{1}{\rho} \frac{\partial \bar{p}}{\partial y}+v \cdot \operatorname{div}(\operatorname{grad} \bar{v})+\left[-\frac{\partial \overline{u^{\prime} v^{\prime}}}{\partial x}-\frac{\partial \overline{v^{\prime 2}}}{\partial y}-\frac{\partial \overline{v^{\prime} w^{\prime}}}{\partial z}\right] \\
\frac{\partial \bar{w}}{\partial t}+\operatorname{div}(\bar{w} \overline{\boldsymbol{U}})=\overline{f_{z}}-\frac{1}{\rho} \frac{\partial \bar{p}}{\partial z}+v \cdot \operatorname{div}(\operatorname{grad} \bar{w})+\left[-\frac{\partial \overline{u^{\prime} w^{\prime}}}{\partial x}-\frac{\partial \overline{v^{\prime} w^{\prime}}}{\partial y}-\frac{\partial \overline{w^{\prime 2}}}{\partial z}\right]
\end{gathered}
$$

The above fluid control equations are not closed, additional turbulence models are needed to make the equations closed, and then the flow properties of the flow field are simulated. The types and numbers of equations introduced for different turbulence models are not the same.

\subsection{Finite volume method}

The finite volume method is a discrete method with high computational efficiency. Most commercial fluid dynamics software uses this method as the core algorithm. The core of the finite volume method is to divide the entire flow field into a series of non-overlapping control bodies by dividing the mesh. By integrating the control equation to be solved on each control body, a discrete equations group can be obtained. The unknown number is the value of the dependent variable at each grid node. In addition, in order to obtain the control volume, it is also necessary to assume the variation of some dependent variables among the grid nodes. It is worth noting that the finite volume method is applied to the subdomain method in the weighted residual method in the selection process of the integral region. In addition, the local approximation method is also used in the finite volume method, so the finite volume method can also be considered as the subdomain method. Add the discrete method [4].

In order not to lose universality, the transport equation of the universal variable for the finite volume method involving the dynamic mesh is given:

$$
\frac{d}{d t} \int_{V} \rho \phi d V+\oint_{S} \rho \phi\left(\vec{U}-\overrightarrow{U_{S}}\right) \cdot \vec{n} d S=\oint_{S} \Gamma_{\phi} \nabla \phi \cdot \vec{n} d S+\int_{V} q_{\phi V} d V
$$

In the above equation, $V$ represents the volume of the control body, $S$ represents the boundary of the control body, $\vec{n}$ is the unit normal vector outside the boundary surface of the control body, $\rho$ is the fluid density, $\vec{U}$ is the fluid velocity vector, $\overrightarrow{U_{s}}$ is the velocity vector of the boundary surface of the control body, and $\Gamma_{\phi}$ is the diffusion coefficient, $q_{\phi V}$ is the source item.

\subsection{Solvers and turbulence models}


The computational fluid dynamics software OpenFOAM is used in this paper. In this paper, PimpleDyMFoam solver is selected for the problem of rotating machinery. The solver is a transient incompressible Newton turbulence solver based on the PIMPLE (PISO-SIMPLE) algorithm. Its built-in AMI boundary can realize data transfer between dynamic and static grid interfaces, which is suitable for the rotary motion model of tidal turbines. At present, the one equation S-A (Spalart-Allmaras) model and the two equation k- $\omega$ SST ( Shear Stress Transport) model have been widely used in the numerical study of rotating machinery such as tidal turbines.[5-6]

The S-A turbulence model was originally proposed for the wall bounded flow in the aerospace field. At present, this model has also been used in the research of turbine rotary machines [7]. As a relatively simple one-equation model, the shock of the S-A model lies in its robustness and speed of calculation. Because only one equation needs to be solved, the convergence speed is fast and the tolerance on the mesh quality, especially in the near wall area, is extremely high. However, the model also has certain deficiencies. The turbulence and time scales in the model are not well defined and cannot be used to predict uniform attenuation, isotropic turbulence, etc.

The k- $\omega$ SST turbulence model was proposed by Menter Scholars in the 1990s. The turbulence model combines the k- $\varepsilon$ and $\mathrm{k}-\omega$ turbulence models and uses the $\mathrm{k}-\omega$ model in the boundary layer region of the fluid near the wall. The k- $\varepsilon$ model is used for the free shear flow in the distance. This model has good simulation ability for the flow field with counter pressure gradient and flow separation, and the calculation time is relatively small. Therefore, the k- $\omega$ SST turbulence will be selected in this paper. The model was numerically simulated [8].

\section{OpenFOAM numerical simulation}

\subsection{Computational domains and boundary conditions}

The computational domain of a two-dimensional turbine is shown in figure1. The entire calculation model is divided into two sub-domains, namely the rectangular outer domain and the circular rotation domain containing the blades.

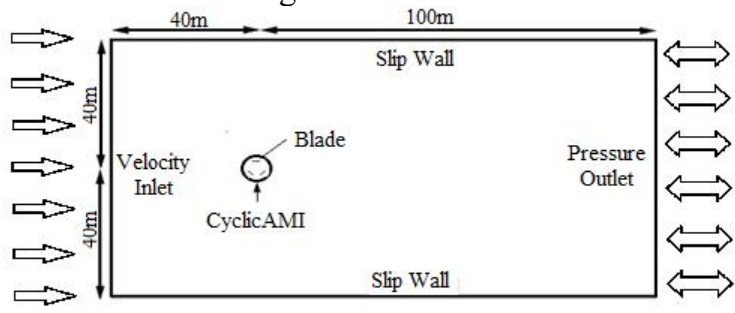

Fig. 1. The calculation domain of tidal turbine.

In the model example, the left side boundary is set as Velocity Inlet, and the velocity is constant; the right side is the Pressure Outlet and the relative pressure is 0; the upper and lower boundaries are set as Slip Wall; the rotation domain and the rectangular domain are connected by the cyclicAMI.

\subsection{Numerical Simulation Method Verification}

To verify the effectiveness of the numerical simulation method proposed in this paper in predicting the vertical axis turbine output power, a three-bladed vertical axis turbine studied 
by Peter et al. was selected as a validation example. The relevant parameters of the turbine are given in table 1 below.

Table 1. Peter turbine model related parameters.

\begin{tabular}{|c|c|c|}
\hline parameter & value & unit \\
\hline Blade airfoil & NACA0020 & \\
\hline Turbine diameter $D$ & 1.0 & $\mathrm{~m}$ \\
\hline Leaves number $Z$ & 3 & \\
\hline Blade chord length $C$ & 0.14 & $\mathrm{~m}$ \\
\hline Leaf length $H$ & 1.0 & $\mathrm{~m}$ \\
\hline Leaf deflection angle $\varphi$ & 0 & $\circ$ \\
\hline Flow velocity $V_{A}$ & 1.0 & $\mathrm{~m} / \mathrm{s}$ \\
\hline Blade installation position $\overline{C_{1}}$ & 0.5 & \\
\hline
\end{tabular}

Figure 2 is a comparison curve between the calculated value and the experimental value of the average energy utilization coefficient $\overline{C_{p}}$ of the turbine. Among them, the peter experimental value is the measured curve given in [9], and the $2 \mathrm{D}$ calculated value is the data curve calculated by the two-dimensional numerical model in this paper. According to the study on the effect of the three-dimensional effect of hydraulic turbines and support structure in the literature [10], when the turbine diameter ratio is 1.0 , the three-dimensional effect will reduce the average energy efficiency of the turbine by about $8 \%$, and the support structure will be reduced by about $9 \%$ average energy utilization. According to the above conclusions, the results of the $2 \mathrm{D}$ simulation in this paper are corrected. In figure 2 , the $3 \mathrm{D}$ correction value represents the result of the modified numerical calculation.

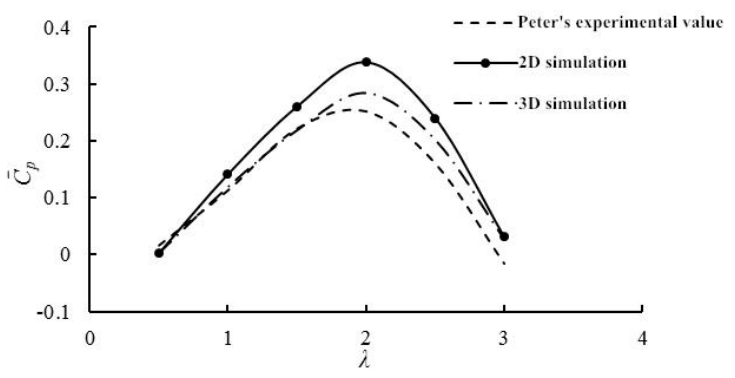

Fig. 2. Average energy efficiency coefficient comparison chart.

From the three groups of comparison curves, it can be seen that the simulated calculation value and the experimental value change trend are similar and both reach the maximum value when the speed ratio is around 2.0. The calculated value is higher than the experimental value. This is because the calculation model can not completely simulate the experimental state of the dragging pond, and does not consider the three-dimensional effect of the blade, the interference of the blade support structure and the spindle, the mechanical friction loss, the measurement error, and the delay of data transmission. influences. Considering the effect of three-dimensional effect and support structure, the absolute error of the measured energy utilization rate after the revised calculation result and the experimental value is $4.16 \%$, which further proves the accuracy of the numerical model adopted in this paper in the study of vertical axis turbines.

\section{Irrelevance analysis of inlet turbulence}


The turbulence degree is defined as Equation 13, and for the fully developed turbulence of the pipe flow, Equation 14 can be used for estimation. When performing CFD simulations, it is usually necessary to pre-estimate the turbulence intensity at the velocity entry.

$$
\begin{gathered}
I=\frac{u^{\prime}}{U}=\frac{\sqrt{\left(u_{x}^{2}+u_{y}^{2}+u_{z}^{2}\right) / 3}}{U} \\
I=0.16 R e_{d}^{-1 / 8}
\end{gathered}
$$

The formula $u^{\prime}$ represents the root mean square of the turbulent pulsation velocity, $u_{\mathrm{x}}$, $u_{\mathrm{y}}{ }^{\prime}, u_{\mathrm{z}}{ }^{\prime}$ are the turbulent fluctuating velocity components, $U$ is the mean velocity of the incoming stream, and $R e_{d}$ is the Reynolds number based on the hydraulic diameter.

In order to investigate the influence of the velocity inlet turbulence on the hydrodynamic performance of the turbine, the velocity inlet turbulence I was selected to be $0.1 \%, 0.5 \%, 1 \%, 5 \%$ and $10 \%$, respectively. The table gives the average energy utilization coefficient values $\overline{C_{p}}$ of the turbine after a stable rotation of the turbine at different speeds of inlet turbulence. The turbine blade tip speed ratio is 2.0.

Table 2. Average energy utilization changes with turbulence.

\begin{tabular}{|l|c|c|c|c|c|}
\hline$I$ & $0.1 \%$ & $0.5 \%$ & $1 \%$ & $5 \%$ & $10 \%$ \\
\hline $\bar{C}_{p}$ & 0.463 & 0.461 & 0.462 & 0.461 & 0.460 \\
\hline
\end{tabular}

According to the data in table 2, it can be concluded that the turbulence at the inlet velocity varies within the range of $0.1 \%-10 \%$, which has little effect on the average energy utilization factor of the turbine, and the turbulence degree has basically no effect on the numerical simulation of the turbine. Taking into account the turbulence range of the turbine running channel, it is recommended to select $5 \%$ of the velocity inlet turbulence in the future numerical simulation.

\section{Conclusion}

This article first introduced the basic theory of CFD method, and then based on OpenFOAM proposed a numerical simulation method of vertical axis turbine, and compared the results with the existing results, verifying the feasibility of the method for the follow-up based on the open source code optimization algorithm to lay the foundation for more efficient calculations. Finally, this paper makes an irrelevance analysis of the influence of inlet turbulence degree, and draws some empirical conclusions, which provides a very valuable reference for future CFD simulation and research of vertical axis tidal turbines.

\section{References}

1. Nabavi Y .Numercal Study of the Duci Shape Effect on the Performance of a Ducted Vertical Axis Tidal Turbine [D].2008Columbia University of British Columbia.

2. GEBRESLASSIE $G$ M, TABOR G R, BELMONT M R. CFD simulations for sensitivity analysis of different parameters to the wake characteristics of tidal turbine[J]. Open Journal of FluidDynamics, 2012, 2(3):56-64. DOI:10.4236/ojfd.2012.230 06.

3. Li Z, Zhang L, Sun K, et al. Numerical simulation of vertical axis Tidal Turbine [J]. Acta Energiae Solaris Sinica, 2011, 32(9):1321-1326. 
4. Toro E F. Riemann solvers and numerical methods for fluid dynamics : a practical introduction[M]// Riemann solvers and numerical methods for fluid dynamics , Springer, 1999.

5. Robertson E, Choudhury V, Bhushan S, et al. Validation of OpenFOAM numerical methods and turbulence models for incompressible bluff body flows[J]. Computers \& Fluids, 2015, 123:122-145.

6. Li W, Yang C, Yu L, et al. Prediction on Hydrodynamic Performances of Propeller by OpenFOAM[C]// ASME 2009, International Conference on Ocean, Offshore and Arctic Engineering, 2009:633-638.

7. Rawlings G W. Parametric characterization of an experimental vertical axis hydro turbine[D]. Vancouver: University of British Columbia, 2008.

8. Menter F R. Two-equation eddy-viscosity turbulence models for engineering applications[J]. Aiaa Journal, 1994, 32(8):1598-1605.

9. Bachant P, Wosnik M. Performance and Near-Wake Measurements for a Vertical Axis Turbine at Moderate Reynolds Number[C]// ASME Fluids Engineering Division Summer Meeting. 2013:V01BT12A005.

10. Li Ye, Calisal S M. Three-dimensional effects and arm effects on modeling a vertical axis tidal current turbine[J]. Renewable Energy, 2010, 35(10):2325-2334. 